\title{
Suppressing the Numerical Cherenkov Instability in FDTD PIC Codes
}

\author{
Brendan B. Godfrey \\ University of Maryland, College Park, Maryland 20742, USA and Lawrence Berkeley \\ National Laboratory, Berkeley, California 94720, USA \\ Jean-Luc Vay \\ Lawrence Berkeley National Laboratory, Berkeley, California 94720, USA
}

Keywords: Particle-in-cell, Finite Difference Time-Domain, Relativistic beam, Numerical stability.

\section{Introduction}

The numerical Cherenkov instability [1] is the most serious numerical instability affecting multidimensional particle-in-cell (PIC) simulations of relativistic particle beams and streaming plasmas [2, 3, 4]. It arises from coupling between numerically distorted electromagnetic modes and spurious beam modes, the latter due to the mismatch between the Lagrangian treatment of particles and the Eulerian treatment of fields [5].

In recent papers we derived and solved electromagnetic dispersion relations for the numerical Cherenkov instability for both finite difference time-domain (FDTD) [6] and pseudo-spectral time-domain (PSTD) [7, 8, algorithms and successfully compared results with those of the Warp simulation code [9]. From the FDTD analysis we developed approximate analytical growth rate expressions for the numerical Cherenkov instability and from them explained the previously observed "magic time-steps" [2, 4] at which instability growth rates decreased significantly. Our PSTD analysis, focused on Haber's Pseudo-Spectral Analytical Time-Domain (PSATD) algorithm [10, 11], provided several methods for suppressing the numerical Cherenkov instability. This was accomplished by a combination of digital filtering at large wave-numbers and improved numerical balancing of transverse electric and magnetic fields at smaller wave numbers. Doing either or both is, of course, mechanically straightforward for PSTD algorithms, because the currents and fields are known in Fourier space and, therefore, can be rescaled easily by the desired k-dependent factors.

In this brief paper we demonstrate that the same can be done economically and with acceptable accuracy for FDTD algorithms without resorting to Fourier transforms. The stabilization process is described in Sec. 2, numerical solutions 
presented in Sec. 3, and sample simulation results given in Sec. 4. Sec. 5 summarizes the paper and suggests further investigations.

\section{Stabilization procedure}

Ref. 6] derives the FDTD dispersion relation for multidimensional PIC codes employing the Esirkepov current-conserving algorithm [12. In the high energy limit it can be written as

$$
C_{0}+n \sum_{m_{z}} C_{1} \csc \left[\left(\omega-k_{z}^{\prime}\right) \frac{\Delta t}{2}\right]+n \sum_{m_{z}} C_{2} \csc ^{2}\left[\left(\omega-k_{z}^{\prime}\right) \frac{\Delta t}{2}\right]=0
$$

with coefficients $C_{0}, C_{1}, C_{2}$ defined in Eqs. (29) - (31) of [6]. Eq. (1) involves sums over numerical aliases, $k_{z}^{\prime}=k_{z}+m_{z} 2 \pi / \Delta z$, for wave numbers aligned with the direction, $z$, of beam propagation. In the limit of vanishingly small time-steps and cell-sizes, Eq. (1) simplifies to $C_{0}=n$, as expected. Thus, all beam resonances in Eq. (1) are numerical artifacts, even $m_{z}=0$. Their interaction with the light modes represented by $C_{0}$ gives rise to the numerical Cherenkov instability. Almost always, the $m_{z}=0,-1$ modes dominate. As noted in the Introduction, instabilities are fastest growing at resonances, which typically occur at large wave numbers (see Figs. 2 and 6 of [6]), where they can be eliminated by digital filtering of the sort described in [2].

The non-resonant form of the numerical Cherenkov instability, although slower growing, is more troublesome, because it often occurs at smaller wave numbers where physical phenomena of interest also occur. We found when analyzing PSATD algorithms that multiplying the fields as seen by the particles at small wave-numbers by k-dependent factors differing from unity by only a few percent was sufficient to cancel the numerical mismatch in $C_{2}$ between transverse electric and magnetic fields and, thereby, nearly eliminate the numerical Cherenkov instability [8]. In principle, the same can be done for FDTD PIC codes. For instance, choosing the multiplier for $E_{x}$ to equal

$$
\frac{S^{B_{y}}}{\left[k_{z}\right]} /\left.\frac{S^{E_{x}}}{[\omega]}\right|_{\omega=k_{z}}
$$

causes $C_{2}$ for $m_{z}=0$ to vanish at $\omega=k_{z}$. Various quantities in Eq. (2) are defined in 6]. Inserting those definitions into Eq. (2) yields

$$
\sin \left(k_{z} \frac{\Delta t}{2}\right) \cos \left(k_{z} \frac{\Delta t}{2}\right) \csc ^{2}\left(k_{z} \frac{\Delta z}{2}\right)\left(k_{z} \frac{\Delta z}{2}\right)\left(\frac{\Delta z}{\Delta t}\right)
$$

for the Galerkin interpolation scheme in [6],

$$
\sin \left(k_{z} \frac{\Delta t}{2}\right) \cos \left(k_{z} \frac{\Delta t}{2}\right) \csc \left(k_{z} \frac{\Delta z}{2}\right)\left(\frac{\Delta z}{\Delta t}\right)
$$


for the Uniform interpolation scheme, and

$$
\sin \left(k_{z} \frac{\Delta t}{2}\right) \cos \left(k_{z} \frac{\Delta t}{2}\right) \cot \left(k_{z} \frac{\Delta z}{2}\right)\left(\frac{\Delta z}{\Delta t}\right)
$$

for a "momentum-conserving" interpolation scheme in which fields are averaged from the usual Yee staggered mesh [13] to a non-staggered mesh before interpolation to the particles.

To apply the multipliers without resorting to Fourier transforms, they are approximated by ratios of fourth-order polynomials in $\sin ^{2}\left(k_{z} \Delta z / 2\right)$, i.e., by rational interpolation functions [14]. The variable $\sin ^{2}\left(k_{z} \Delta z / 2\right)$ is chosen for its simple $(1,-2,1)$ stencil in $z$, and fourth-order is chosen as a reasonable compromise between accuracy and economy. The nine interpolation points from which the polynomial coefficients are determined are spaced uniformly between 0 and 1 in $\sin ^{2}\left(k_{z} \Delta z / 2\right)$. Better choices for the interpolation points may exist. As in the earlier papers, Mathematica [15] is used to solve the dispersion relation; its RationalInterpolation function conveniently provides the desired polynomial coefficients ${ }^{1}$ Fig. 1 displays $\Psi_{E}$, the numerator polynomial, $\Psi_{B}$, the denominator polynomial, and the ratio of the two, the rational interpolation function for, in this case, Eq. 4 with $\Delta t / \Delta z=0.9$ and $\beta_{z}=1 / 8$ (i.e., with the Cole-Karkkainen field-solver [17, 18, 19]). The rational interpolation function is accurate to $10^{-6}$ except for the largest values of $k_{z} \Delta z$ shown in the figure, where the accuracy still is better than $10^{-5}$.

The numerator, $\Psi_{E}$, of the rational interpolation function can be applied to $E_{x}$ before interpolation to the particles by repeated applications of the $(1,-2,1)$ stencil, and the denominator, $\Psi_{B}$, by repeated application of its inverse, in other words, by tri-diagonal matrix inversion. However, because some digital filtering is needed anyway, it is simplier to apply only the numerator polynomial to $E_{x}$, and the denominator polynomial to $B_{y}$ and $E_{z}$, in effect using the denominator as a digital filter. (In three dimensiions $\Psi_{E}$ is applied to both transverse $E$ fields, and $\Psi_{B}$ to all other fields.) This approach is implemented in WARP.

\section{Numerical solutions}

This section presents solutions of the complete FDTD-Esirkepov linear dispersion relation, Eq. (11) of [6] with interpolation function $S^{E_{x}}$ multiplied by $\Psi_{E}$, and $S^{B_{y}}$ and $S^{E_{z}}$ multiplied by $\Psi_{B}$. Digital filtering is as in Eq. (37) of [6] but with the exponent " 16 " replaced by " 4 ",

$$
\cos ^{4}\left(k_{z} \frac{\Delta z}{2}\right)\left(5-4 \cos ^{2}\left(k_{z} \frac{\Delta z}{2}\right)\right)^{2} \cos ^{4}\left(k_{x} \frac{\Delta x}{2}\right)\left(5-4 \cos ^{2}\left(k_{x} \frac{\Delta x}{2}\right)\right)^{2}
$$

${ }^{1}$ Software to calculate these coefficients is available in Computable Document Format [16] at http://hifweb.lbl.gov/public/BLAST/Godfrey/ 
equivalent to two passes each of a bilinear filter and a compensation filter [2]. Thus, much less digital filtering is applied. Cubic interpolation is employed on a two-dimension, rectangular, periodic, $128 \times 128$ mesh with cell size 0.3868 . Linear interpolation also was tried but did not produce uniformly good stability results.

Maximum numerical instability growth rates for a beam of energy $\gamma=130$ are shown as a function of $v \Delta t / \Delta z$ in Fig. 2 (Note that the determination of $\Psi_{E}$ and $\Psi_{B}$ breaks down at $0.756<v \Delta t / \Delta z<0.764$, and this narrow region has been excised from the Figs. 2 and 3.) Also shown are growth rates obtained from WARP simulations; agreement is good. These growth rates are much smaller than those obtained without the rational interpolation factors but with otherwise identical parameters, given by the curves labeled "GalerkinCK" and "Uniform-CK" in Fig. 16 of [7. In fact, the growth rates in Fig. 2 are comparable to those labeled "PSATD (c)" in Fig. 16 of [7, which is not surprising: Both are based on zeroing $\mathrm{C} 2$ at $\omega=k_{z}$. Although not shown here, the dispersion relation also has been solved for variants of the Fig. 2 parameters with (1) no digital filtering (apart from $\Psi_{B}$ ), which roughly doubles growth rates at large $v \Delta t / \Delta z ;(2) \Psi_{E} / \Psi_{B}$ applied to $E_{x}$ only, which roughly triples growth rates at large $v \Delta t / \Delta z$; and (c) not employing the compensation filter, which moderately reduces growth rates, especially at large $v \Delta t / \Delta z$. Of course, physical results at small wave numbers also may be filtered modestly in this last case [2].

Because the scaling procedure described in this paper is tuned for infinite $\gamma$, it works less well at only modestly relativistic beams, as illustrated in Fig. 3 for $\gamma=3$. Based on this and other computations, peak growth rates appear to scale here very roughly as $\gamma^{-1 / 2}$. Tuning the procedure for moderate $\gamma$ is more difficult, because the dispersion relation is more complicated. Nonetheless, the numerical instability growth rates depicted in Fig. 3 probably are acceptable for most purposes. At still smaller beam energies, the well known $m_{z}=-1$ quasione-dimensional, electrostatic numerical instability [20, 21] dominates. It can be suppressed by using any field interpolation algorithm that offsets $E_{z}$ by $\triangle z / 2$ relative to the charge density $\varrho$ (or $\mathbf{W}$ in the Esirkepov current algorithm) and interpolates it with a spline one order lower in z relative to $\varrho$ or to $\mathbf{W}$ [22, 23], such as the Galerkin algorithm.

\section{Application to laser plasma acceleration simulations}

In a laser plasma accelerator (LPA), a laser pulse is propagated through a plasma, creating a wake of very strong electric fields of alternating polarity [24]. An electron beam injected with the appropriate phase thus can be accelerated to high energy in a distance much shorter than those for conventional acceleration techniques [25]. As a verification that the theory developed in this paper applies to the modeling of LPAs, series of three dimensional simulations of a $100 \mathrm{MeV}$ class LPA stage were performed, focusing on the plasma wake formation. The velocity of the wake in the plasma corresponds to $\gamma \simeq 13.2$, and the simulations were performed in a boosted frame of $\gamma_{f}=13$. The LPA simulations with 
parameters leading to Figs. 15 and 16 in [6] were repeated using the procedure discussed in this paper, providing results such as those in Fig. 4. (Note that both these and the simulation sweeps in [6] used the digital filter with one pass each of a bilinear filter and a compensation filter, equivalent to the square root of the expression in Eq. 6, rather than the expression in Eq. (37) of [6].) Energy conservation was excellent when the rational interpolation multipliers were used.

Additionally, three dimensional $\gamma=13$ LPA simulations of the sort described in [26] were performed to validate the stabilization procedure. Fig. 5 records the accelerated electron beam RMS radius. Six simulations used $\Delta t / \Delta z=0.99$; those employing the rational interpolation multipliers behaved as desired, while those that did not produced meaningless results due to the numerical Cherenkov instability. An almost instability-free uniform interpolation simulation at the $v \Delta t / \Delta z=0.5$ "magic time step" is provided for comparison. All four stable simulations provided essentially identical results, including the evolution of beam energy (not shown), emittance (not shown), and radius.

\section{Conclusion}

This paper presents a straightforward approach for greatly reducing numerical Cherenkov instability growth rates in FDTD-Esirkepov PIC simulations of relativistic beams and streaming plasmas. Moreover, sample simulations indicate that this approach is economical, requires minimal additional digital filtering, and apparently has no adverse effect on physical results at wavelengths long compare to the simulation axial cell size. Although derived for highly relativistic flows, it works reasonably well down to $\gamma$ of order 3 , below which the numerical Cherenkov instability ceases to be the dominant numerical effect.

While this approach seems quite promising, possibly even better procedures may be possible, including some adaptable from approaches already demonstrated for PSATD PIC algorithms 7, 8. We hope to explore such alternatives in the near future. We also anticipate generalizing these procedures to FDTD simulations not employing the Esirkepov algorithm, and to a wider range of Maxwell solvers.

\section{Acknowledgments}

We wish to thank Irving Haber for suggesting this collaboration and for helpful recommendations. We also are indebted to David Grote for assistance in using the code WARP. This work was supported in part by the Director, Office of Science, Office of High Energy Physics, U.S. Dept. of Energy under Contract No. DE-AC02-05CH11231 and the US-DOE SciDAC ComPASS collaboration, and used resources of the National Energy Research Scientific Computing Center.

This document was prepared as an account of work sponsored in part by the United States Government. While this document is believed to contain correct information, neither the United States Government nor any agency thereof, nor The Regents of the University of California, nor any of their employees, nor 
the authors makes any warranty, express or implied, or assumes any legal responsibility for the accuracy, completeness, or usefulness of any information, apparatus, product, or process disclosed, or represents that its use would not infringe privately owned rights. Reference herein to any specific commercial product, process, or service by its trade name, trademark, manufacturer, or otherwise, does not necessarily constitute or imply its endorsement, recommendation, or favoring by the United States Government or any agency thereof, or The Regents of the University of California. The views and opinions of authors expressed herein do not necessarily state or reflect those of the United States Government or any agency thereof or The Regents of the University of California.

\section{References}

\section{References}

[1] B. B. Godfrey, Numerical cherenkov instabilities in electromagnetic particle codes, Journal of Computational Physics 15 (4) (1974) 504-521.

[2] J.-L. Vay, C. G. R. Geddes, E. Cormier-Michel, D. P. Grote, Numerical methods for instability mitigation in the modeling of laser wakefield accelerators in a lorentz-boosted frame, Journal of Computational Physics 230 (15) (2011) 5908-5929. doi:10.1016/j.jcp.2011.04.003.

[3] L. Sironi, A. Spitkovsky, private Communication (2011).

[4] X. Xu, P. Yu, S. F. Martins, F. Tsung, V. K. Decyk, J. Vieira, R. A. Fonseca, W. Lu, L. O. Silva, W. B. Mori, Numerical instability due to relativistic plasma drift in em-pic simulations, Computer Physics Communications 184 (2013) 2503-2514.

[5] B. B. Godfrey, Canonical momenta and numerical instabilities in particle codes, Journal of Computational Physics 19 (1) (1975) 58-76.

[6] B. B. Godfrey, J.-L. Vay, Numerical stability of relativistic beam multidimensional pic simulations employing the esirkepov algorithm, Journal of Computational Physics 248 (2013) 33-46. doi:10.1016/j.jcp.2013.04. 006.

[7] B. B. Godfrey, J.-L. Vay, I. Haber, Numerical stability analysis of the pseudo-spectral analytical time-domain pic algorithm, Journal of Computational Physics 258 (2014) 689-704. doi:10.1016/j.jcp.2013.10.053.

[8] B. B. Godfrey, J.-L. Vay, I. Haber, Numerical stability improvements for the pseudo-spectral em pic algorithm (2013).

[9] D. Grote, A. Friedman, J.-L. Vay, I. Haber, The warp code: modeling high intensity ion beams, in: AIP Conference Proceedings, no. 749, 2005, pp. $55-58$. 
[10] I. Haber, R. Lee, H. Klein, J. Boris, Advances in electromagnetic simulation techniques, in: Proc. Sixth Conf. Num. Sim. Plasmas, Berkeley, CA, 1973, pp. $46-48$.

[11] J.-L. Vay, I. Haber, B. B. Godfrey, A domain decomposition method for pseudo-spectral electromagnetic simulations of plasmas, Journal of Computational Physics 243 (2013) 260-268. doi:10.1016/j.jcp.2013.03.010.

[12] T. Esirkepov, Exact charge conservation scheme for particle-in-cell simulation with an arbitrary form-factor, Computer Physics Communications 135 (2) (2001) 144-153.

[13] K. Yee, Numerical solution of initial boundary value problems involving maxwell's equations in isotropic media, IEEE Transactions on Antennas and Propagation AP14 (3) (1966) 302-307.

[14] F. Larkin, Some techniques for rational interpolation, The Computer Journal 10 (1967) 178-187.

[15] Mathematica, version nine (2012). URL http://www.wolfram.com/mathematica/

[16] Computable document format (cdf) (2012). URL http://www. wolfram.com/cdf/

[17] J. Cole, A high-accuracy realization of the yee algorithm using nonstandard finite differences, IEEE Transactions on Microwave Theory and Techniques 45 (6) (1997) 991-996.

[18] J. Cole, High-accuracy yee algorithm based on nonstandard finite differences: New developments and verifications, IEEE Transactions on Antennas and Propagation 50 (9) (2002) 1185-1191. doi:10.1109/TAP. 2002. 801268 .

[19] M. Karkkainen, E. Gjonaj, T. Lau, T. Weiland, Low-dispersion wake field calculation tools, in: Proc. International Computational Accelerator Physics Conference, Chamonix, France, 2006, pp. 35-40.

[20] A. B. Langdon, Nonphysical modifications to oscillations, fluctuations, and collisions due to space-time differencing, in: Proceedings of the fourth conference on numerical simulation of plasmas, 1970, pp. 467-495.

[21] H. Okuda, Nonphysical instabilities in plasma simulation due to small debye length, in: Proceedings of the fourth conference on numerical simulation of plasmas, 1970, pp. 511-525.

[22] H. R. Lewis, Variational algorithms for numerical simulation of collisionless plasma with point particles including electromagnetic interactions, Journal of Computational Physics 10 (3) (1972) 400-419. 
[23] A. B. Langdon, Energy-conserving plasma simulation algorithms, Journal of Computational Physics 12 (2) (1973) 247-268.

[24] T. Tajima, J. Dawson, Laser electron-accelerator, Physical Review Letters 43 (4) (1979) 267-270.

[25] W. P. Leemans, B. Nagler, A. J. Gonsalves, C. Toth, K. Nakamura, C. G. R. Geddes, E. Esarey, C. B. Schroeder, S. M. Hooker, Gev electron beams from a centimetre-scale accelerator, Nature Physics 2 (10) (2006) 696-699. doi:10.1038/nphys418.

[26] J.-L. Vay, C. G. R. Geddes, E. Esarey, C. B. Schroeder, W. P. Leemans, E. Cormier-Michel, D. P. Grote, Modeling of 10 gev-1 tev laser-plasma accelerators using lorentz boosted simulations, Physics of Plasmas 18. doi: $10.1063 / 1.3663841$. 


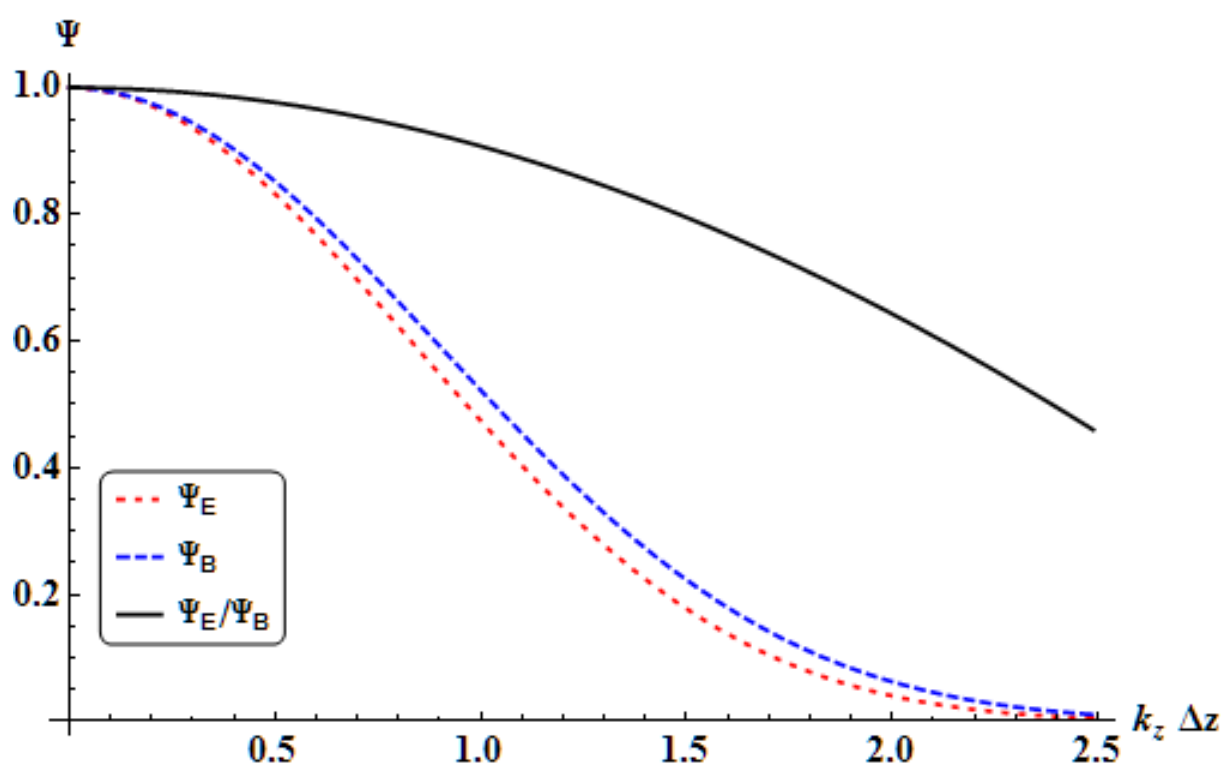

Figure 1: Field multipliers $\Psi$ for Uniform interpolation scheme with $\Delta t / \Delta z=0.9$. 


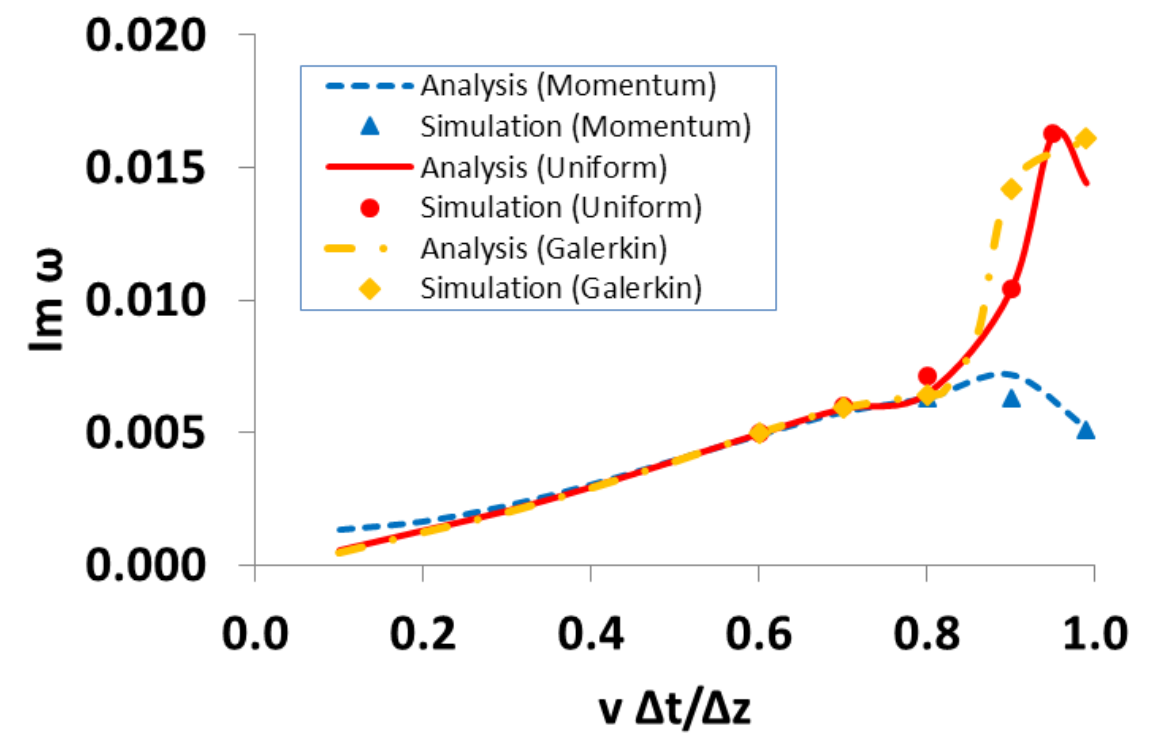

Figure 2: Maximum numerical instability growth rates observed in WARP and calculated from the numerical dispersion relation for $\gamma=130$. 


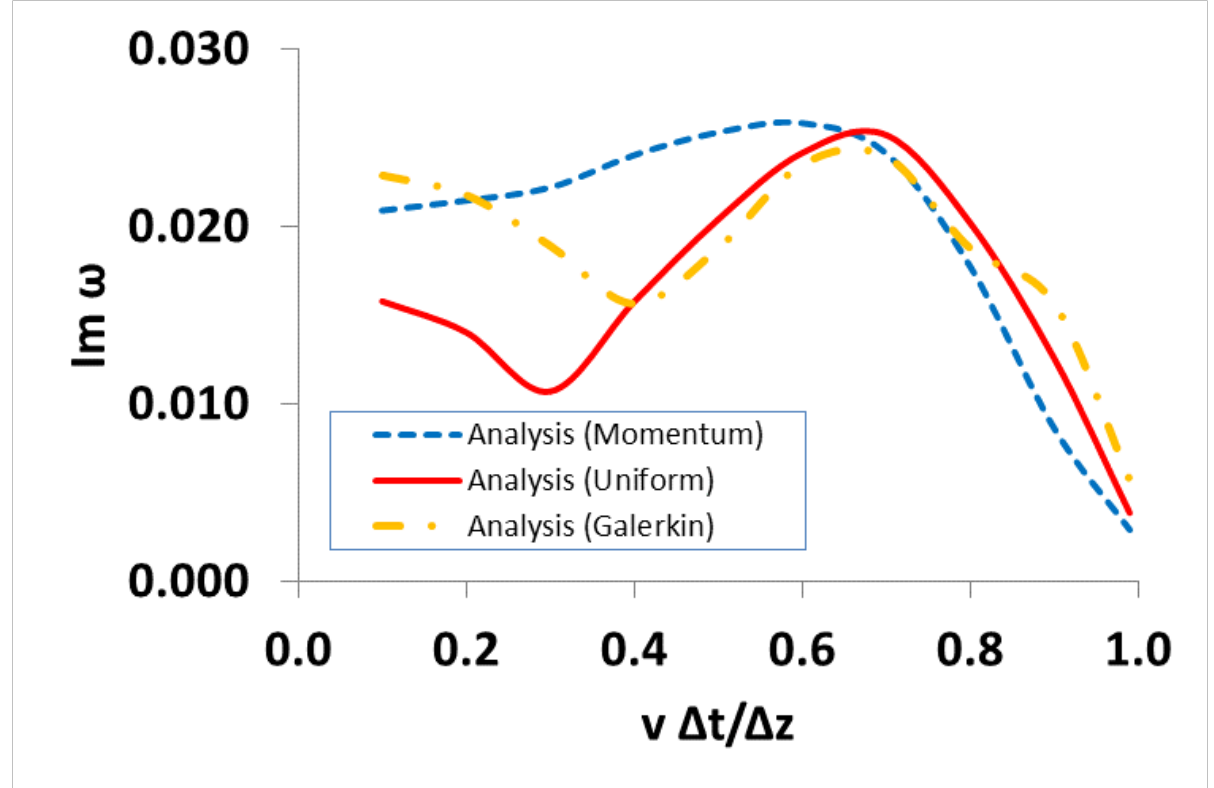

Figure 3: Maximum numerical instability growth rates calculated from the numerical dispersion relation for $\gamma=3$. 


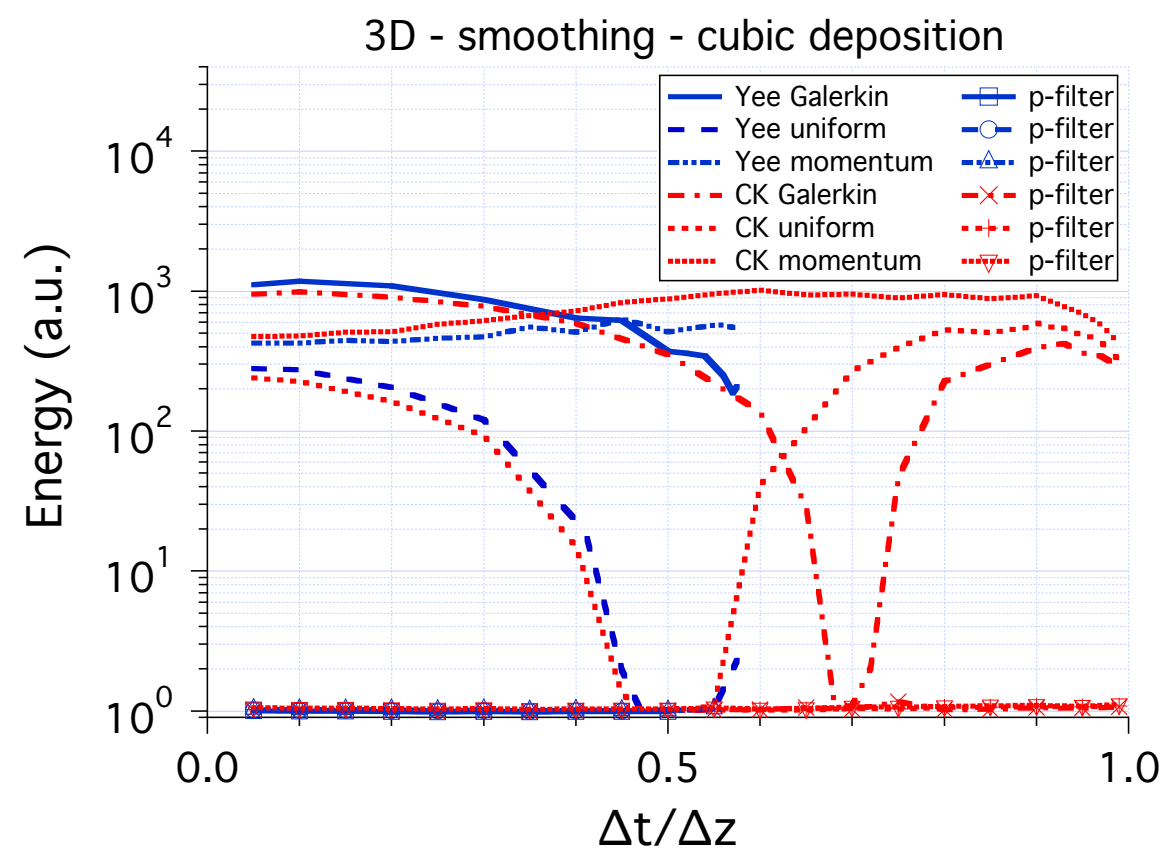

Figure 4: Field energy relative to stable reference level vs $\Delta t / \Delta z$ from three dimensional WARP LPA simulations at $\gamma=13$, using Galerkin, uniform, and momentum-conserving field interpolation with either standard ("Yee") or Cole-Karkainnen ("CK") field solvers. The relative energy is essentially unity for all instances in which the rational interpolation function ("p-filter") is applied. 


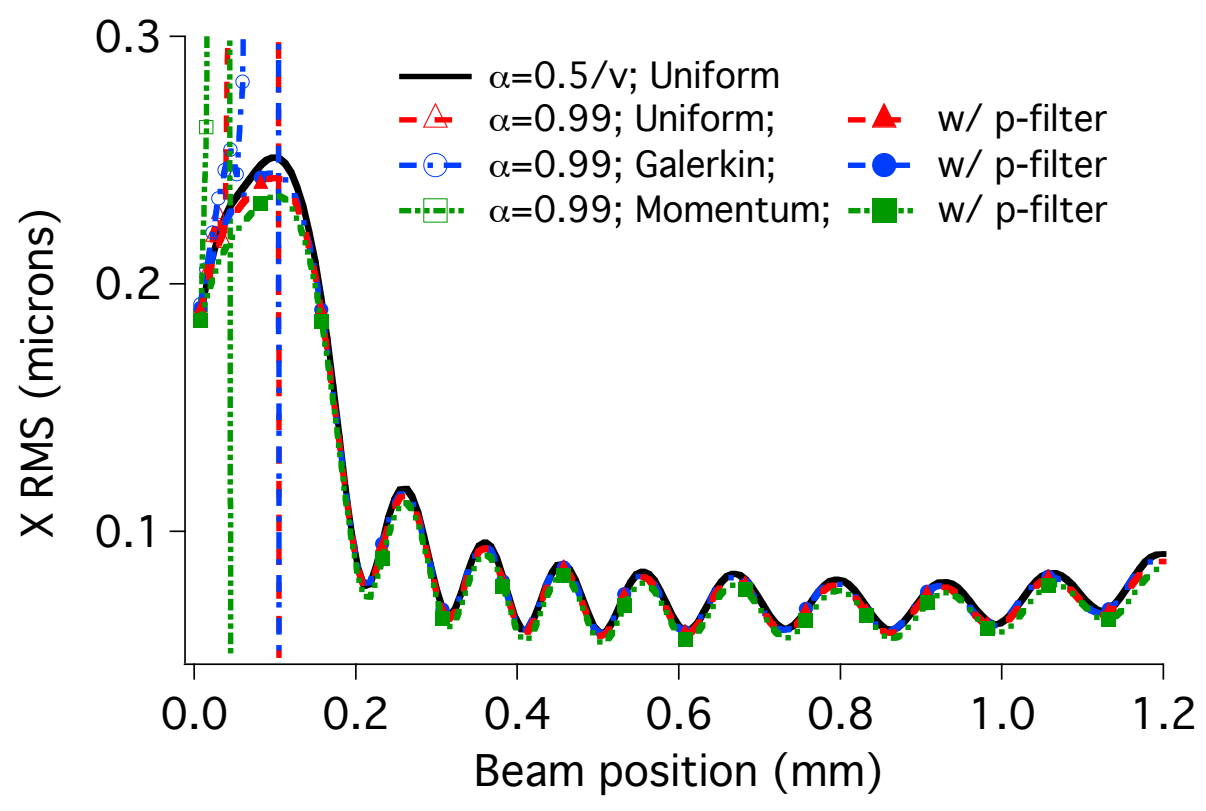

Figure 5: Accelerated electron beam RMS radius from $\gamma=13$ LPA simulations. Six runs used $\alpha \equiv \Delta t / \Delta z=0.99$; those employing the rational interplation multipliers ("p-filter") behaved well, while those that did not produced meaningless results. An almost instability-free uniform interpolation simulation at the $v \Delta t / \Delta z=0.5$ "magic time step" is provided for comparison. 